\title{
Variations de la température de l'eau des étangs en période froide à Lubumbashi (R.D. Congo) et implications pour la production des poissons
}

\author{
Lwamba Balimwacha Jules. ${ }^{1^{*}}$, Katim Mwin A Mpal Antoine ${ }^{1}$, Kiwaya Alunga Trésor ${ }^{1}$, Ipungu \\ Lushimba Raymond ${ }^{2}$, Nyongombe Utshudienyema Nathan ${ }^{3}$ \\ 1 Université de Lubumbashi, Faculté des Sciences Agronomiques, Département de Zootechnie, Unité de Recherche \\ en Aquaculture «U.R.A. », Lubumbashi, BP 1825 RD Congo ; \\ 2Université de Lubumbashi, Faculté de Médecine vétérinaire, Département de Zootechnie, Unité de Recherche en \\ Aquaculture «U.R.A. », Lubumbashi, BP 1825 RD Congo ; \\ 3 Université Pédagogique Nationale, Kinshasa, RD Congo. \\ *Correspondance : Tel : +243 (0) 816510279 ; +243 (0) 997021151 ; e-mail : juleslwamba@gmail.com
}

Original submitted in on 30th April 2015. Published online at www.m.elewa.org on $30^{\text {th }}$ June 2015

http://dx.doi.org/10.4314/jab.v90i1.5

\begin{abstract}
RESUME
Objectif: Cette étude a été initiée en vue de mettre en évidence les variations de température de l'eau des étangs en période froide à Lubumbashi et leurs implications sur la croissance et la reproduction des poissons.

Méthodologie et résultats: La température de l'eau a été prélevée à l'aide de 3 thermomètres maxi minima hors de l'eau (à $50 \mathrm{~cm}$ ) et à différentes profondeurs (15 et $100 \mathrm{~cm}$ dans l'eau). Les données collectées ont été traitées suivant une analyse de la variance avec test post hoc de student. Les résultats obtenus révèlent que la température moyenne journalière des étangs pendant la période d'étude était de 19,4 \pm $2^{\circ} \mathrm{C}$, différente de la température minimale seuil de $22^{\circ} \mathrm{C}(\mathrm{p}<0,001)$. S'agissant des variations journalières, les températures de $19^{\circ} \mathrm{C}$ sont enregistrées à partir de 6 heures du matin contre celles de $22^{\circ} \mathrm{C}$ à 17 heures et $19^{\circ} \mathrm{C}$ à 21 heures. Par ailleurs, la température obtenue hors l'eau des étangs a été supérieure aux températures similaires enregistrées à $15 \mathrm{~cm}$ et $100 \mathrm{~cm}$ de profondeur.

Conclusion et application : La mise en évidence de la courbe de variation de température en période froide à Lubumbashi est cruciale pour la mise en place des systèmes de chauffage d'étangs peu couteux en vue d'intensifier durablement la pisciculture. Nous suggérons que des études évaluent à court terme la réponse de différentes espèces de poissons élevées dans la zone d'étude à ces variations de température en période froide.
\end{abstract}

MOTS CLES : Température, eau, période froide, pisciculture, Lubumbashi.

\section{ABSTRACT}

Temperature of the pond water during the cold period in Lubumbashi (DR Congo): variations and implications on growth and reproduction of fish

Objective: This study was initiated to highlight the pond water temperature changes during cold season in Lubumbashi and to evaluate their implications on growth and reproduction of fish. 


\section{Lwamba et al. J. Appl. Biosci. Variations de la température de l'eau des étangs en période froide à Lubumbashi (R.D. Congo) et implications pour la production des poissons}

Methods and Results: The temperature of the water was measured using 3 maxi mini thermometers, outside water (at $50 \mathrm{~cm}$ ) and at different depths $(15$ and $100 \mathrm{~cm}$ in water). The collected data were processed on an analysis of variance with post hoc test of student. The results showed that the average daily temperature of the pond during the study period was $19.4 \pm 2^{\circ} \mathrm{C}$, different from minimum threshold temperature of $22^{\circ} \mathrm{C}(p<0.001)$. Regarding the daily variations, temperature of $19^{\circ} \mathrm{C}$ was recorded from 6 am against $22^{\circ} \mathrm{C}$ at $5 \mathrm{pm}$ and $19^{\circ} \mathrm{C}$ at $9 \mathrm{pm}$. Furthermore, the temperature obtained outside pond water was higher than temperatures recorded at $15 \mathrm{~cm}$ and $100 \mathrm{~cm}$ deep. Conclusion and Application: The detection of temperature change curve during cold season in Lubumbashi is crucial to the development of inexpensive heating systems of ponds to intensify sustainable fish farming. We suggest that short-term studies assess the response of different fish species product in the study area for these temperature variations in the cold period.

Keywords: Temperature, Water, Cold Period, Pond, Lubumbashi

\section{INTRODUCTION}

La pêche en Afrique est une activité essentielle aussi bien pour la sécurité alimentaire que pour le développement économique. Elle assure la subsistance de 10 millions et nourrit 20 millions de personnes, soit $1 / 5$ des africains, apportant $22 \%$ de leurs besoins aux protéines animales (Spore, 2013; Valeta et al., 2013). Mais, l'actuelle stagnation des captures oblige l'Afrique à développer sa filière aquacole, si elle ne veut pas accroître ses importations de poissons (Spore, 2013 ; Nwano et al., 2014). Toutefois, cette filière est butée à plusieurs contraintes naturelles, entre autres la salinité et la température (Nwano et al., 2014). S'agissant de la température de l'eau, elle intervient directement sur la vie des poissons et la croissance et l'activité du poisson dépendent de la température de son corps (Halwart \& Gupta, 2010). En effet, la température du corps du poisson est à peu près la même que celle de l'eau et elle varie en fonction de celle-ci (Coche \& Muir, 1995 ; Caudron, 2008). La température de l'eau accroît les vitesses des réactions chimiques et biochimiques d'un facteur 2 à 3 fois pour une augmentation de 10 unités $\left({ }^{\circ} \mathrm{C}\right)$ (Caudron, 2008). D'une manière générale, une élévation de température jusqu'à un niveau optimal accélère la croissance des poissons alors qu'une baisse de celle-ci provoque l'effet inverse (De Villers et al., 2005 ; Omasaki et al., 2013). L'eau d'un étang évolue selon les saisons et les localisations géographiques de 2 à $30^{\circ} \mathrm{C}$. Mais, chose moins connue, les conditions de température d'un cours d'eau peuvent également varier de 4 à $10^{\circ} \mathrm{C}$ au cours d'une même journée; ce qui justifie les différences d'activité des poissons que l'on observe au cours d'une journée de pêche (Dequé, 2008). En fonction d'espèces, du stade de vie (embryon, larve, juvéniles ou adultes) et de l'état physiologique (maturité sexuelle), les exigences écologiques vis-à-vis de la température ne sont pas les mêmes (Aktas et al., 2003 ; Dequé, 2008 ; El Gamal, 2009). Les tilapias, notamment Oreochromis niloticus, sont caractérisés par un cycle de reproduction assez court (Mukasikubwabo, 1990). Leur optimum de développement se situe à des températures supérieures à $20^{\circ} \mathrm{C}$ et allant jusqu'à plus de $30^{\circ} \mathrm{C}$ (Huet, 1970). La température est un élément primordial à la base de la notion de gestion et dont toute intervention sur l'étang devra l'intégrer (Caudron, 2008). L'O. niloticus est généralement élevé dans les eaux chaudes industrielles en régions tempérées (Kestemont et al., 1989). Par ailleurs, dans les régions subtropicales africaines, un arrêt de croissance est observé pendant l'hiver alors que la reprise de croissance n'intervient que lorsque la température augmente (Bruton \& Allanson, 1974). Daget $(1952 ; 1956)$ relève jusqu'à six mois d'arrêt de croissance en saison sèche dans le Delta Central du Niger. Dans le lac Tchad, Durand (1978) et Hopson (1972) observent un arrêt de croissance de cinq mois chez Alestes baremoze en période froide. Dans le Sud-est africain, la plupart des travaux font état d'une 


\section{Lwamba et al. J. Appl. Biosci. Variations de la température de l'eau des étangs en période froide à Lubumbashi (R.D. Congo) et implications pour la production des poissons}

reprise de croissance de poissons pendant le printemps austral (Balon \& Coche, 1974). II est possible de rattacher à ces milieux les lacs d'altitude de Madagascar étudiés par Moreau (1979). Au Rwanda, il s'est avéré que l'élevage en étangs d'O. niloticus a suscité des problèmes de production d'alevins (10 à 30 alevins/femelle/mois) en saison sèche à la suite des brusques changements de température de l'eau $\left(19\right.$ à $\left.28^{\circ} \mathrm{C}\right)$ (Mukasikubwabo, 1990). Pourtant, à Lubumbashi en République Démocratique du Congo, une

\section{MATERIEL ET METHODES}

Milieu d'étude : La présente étude a été réalisée à la station de l'Unité de Recherche en Aquaculture « U.R.A.» de l'Université de Lubumbashi. L'URA est interruption de production des 0 . niloticus est également observée pendant la saison froide. L'objet de cette étude est de déterminer les variations de température de l'eau en étang pendant la saison froide à Lubumbashi. Nous testons les hypothèses selon lesquelles la période froide à Lubumbashi entraine une baisse de température, en dépit de l'insolation (1) et que le milieu ambiant influence la variation de la température de l'eau dans un étang, aussi bien en surface qu'en profondeur (2).

localisée dans le site piscicole du Jardin Zoologique $\left(\mathrm{S} 11^{\circ} 40^{\prime} 08^{\prime \prime}\right.$ et E27 ${ }^{\circ} 28^{\prime} 311^{\prime \prime}$ ) (figure 1).
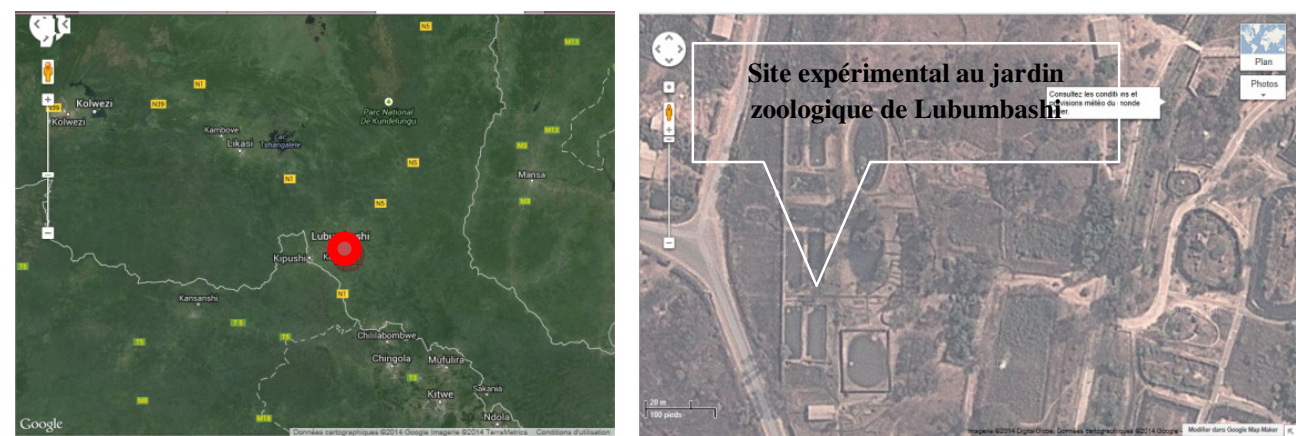

Figure 1: La ville de Lubumbashi (point rouge à gauche) et le site expérimental au sein du jardin zoologique de Lubumbashi (à droite) (Google earth, 2014).

Actuellement, trois espèces de poissons y font objet de recherche, notamment le tilapia du Nil (Oreochromis niloticus) et le poisson-chat africain (Clarias ngamensis et Clarias gariepinus). Les étangs dont la profondeur varie entre 120 et $80 \mathrm{~cm}$, sont alimentés par la rivière Lubumbashi par le biais d'un canal de dérivation qui traverse ledit Jardin. Le climat de la ville de Lubumbashi est caractérisé par un régime pluviométrique contrasté entre une saison des pluies (de Novembre à Mars), une saison sèche (de Mai à
Septembre) et une période de transition (Octobre et Avril) (Leblanc \& Malaisse, 1978). La température moyenne annuelle est de $20^{\circ} \mathrm{C}$.. Ainsi, Octobre et Novembre sont les mois les plus chauds avec une moyenne des maxima journaliers de $32^{\circ} \mathrm{C}$ et une température moyenne mensuelle de $23^{\circ} \mathrm{C}$. Par contre, le mois de Juillet est le mois le plus froid (figure 2) avec la moyenne des minima journaliers de $8^{\circ} \mathrm{C}$, la température moyenne mensuelle étant de $17^{\circ} \mathrm{C}$. L'humidité relative atteint 48\% (Leblanc et al., 1978). 


\section{Lwamba et al. J. Appl. Biosci. Variations de la température de l'eau des étangs en période froide à Lubumbashi (R.D. Congo) et implications pour la production des poissons}

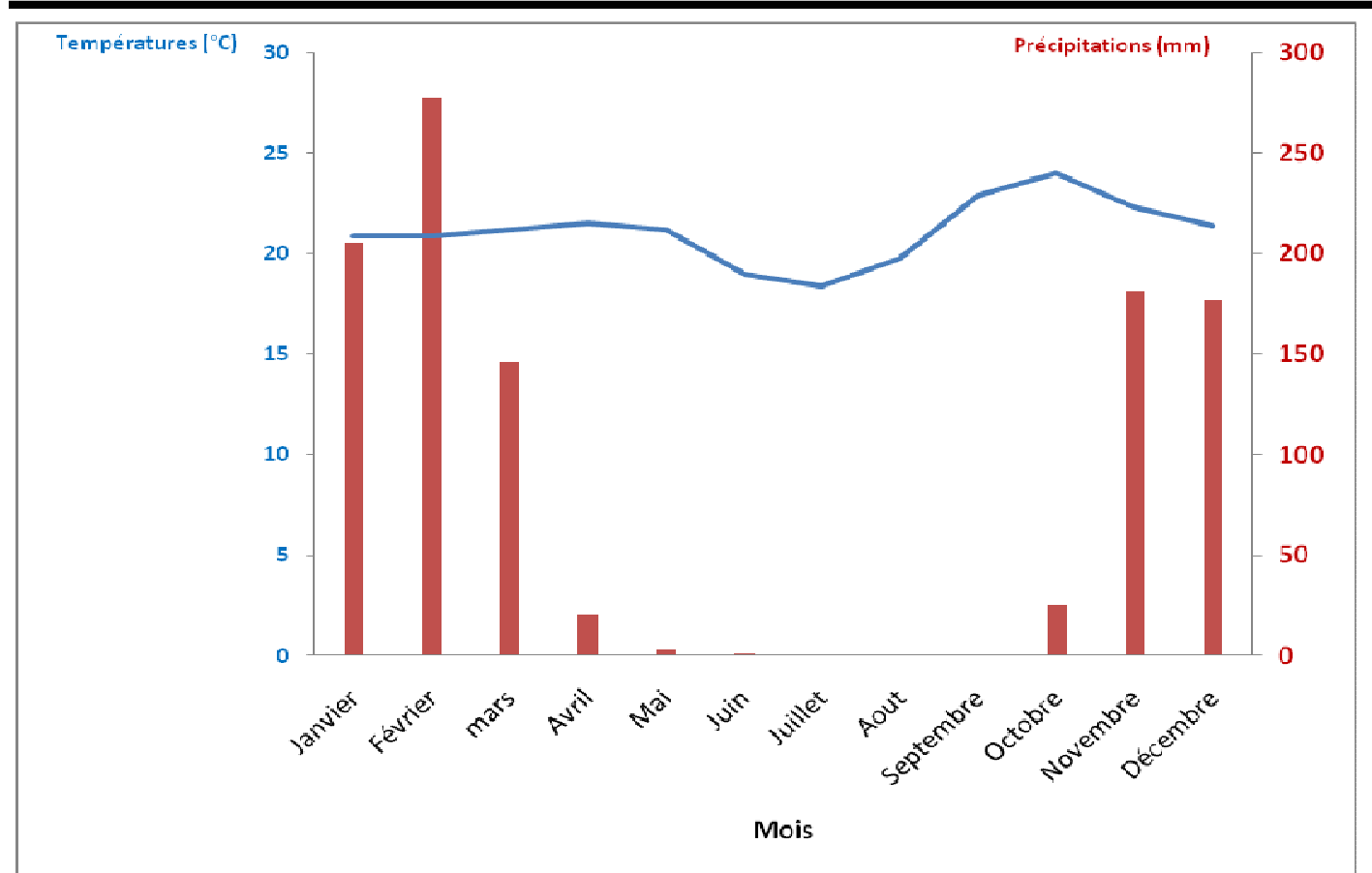

Figure 2 : Diagramme ombrothermique de Lubumbashi en 2011. Station de Luano, altitude $1298 \mathrm{~m}$, longitude $28^{\circ} 29^{\prime} \mathrm{E}$, latitude $11^{\circ} 40^{\prime} \mathrm{S}$. Source : Mettelsat /Katanga 2015.

Matériels et méthodes : La température de l'eau des étangs a été prélevée à différentes profondeurs (15 et $100 \mathrm{~cm})$, en comparaison à celle hors de l'eau $(50 \mathrm{~cm}$ de la surface de l'étang), pour la détermination de la courbe de variation journalière de la température de l'eau dans l'étang en période froide. Pour éviter le biais dans le prélèvement de données, les thermomètres ont été fixés à un même support en bois plongé dans l'eau à différentes profondeurs (Coche \& Muir, 1999). En effet, sur un même support en bois d'environ $2 \mathrm{~m}$, un thermomètre était situé à $50 \mathrm{~cm}$ au dessus de la lame d'eau, un deuxième à $15 \mathrm{~cm}$ de profondeur et le troisième à $100 \mathrm{~cm}$ de profondeur. Le support en bois était légèrement incliné, tel que recommandé par Coche \& Muir (1999), et fixé au milieu de l'étang. La

\section{RESULTATS}

Variation journalière de température de l'eau: La température moyenne journalière des étangs était de $19,4 \pm 2^{\circ} \mathrm{C}$ pendant la période d'étude. En la comparant avec la température de $22^{\circ} \mathrm{C}$ qui est le seuil minimum de référence, il a été constaté une différence significative $(p<0,001)$ (figure 3$)$. Le monitoring de 10 jours successifs de température a montré qu'il existe deux périodes des fluctuations importantes. D'une part, la période allant de $22 \mathrm{~h}$ à 10 heures enregistre des collecte des données a été réalisée du 03 au 12 juillet 2011 à l'aide de 3 thermomètres maxi minima (Coche \& Muir, 1995 ; 1999). Le choix de cette période d'étude est justifié par le fait que le mois de juillet enregistre des basses des températures dont il était indispensable de caractériser leur variation et d'évaluer si elles affectent la température d'eau des étangs. La lecture des températures extrêmes se faisait une fois le matin (à 8 h00') et les curseurs étaient ramenés à zéro. Le seul paramètre observé dans cette étude était la température. L'analyse de la variance, avec test post hoc de Student, a été réalisée avec le logiciel SPSS 16.0. La valeur de $p<0,05$ était considérée comme significative.

basses températures de $17^{\circ} \mathrm{C}$ à $19^{\circ} \mathrm{C}$ bien que la température la plus basse ait été observée à 6 heures du matin. D'autre part, les températures supérieures à $19^{\circ} \mathrm{C}$ sont enregistrées à partir de 6 heures du matin. Cette augmentation de la température atteint un pic de $22^{\circ} \mathrm{C}$ à 17 heures et redescend pour atteindre $19^{\circ} \mathrm{C}$ à 21 heures. 


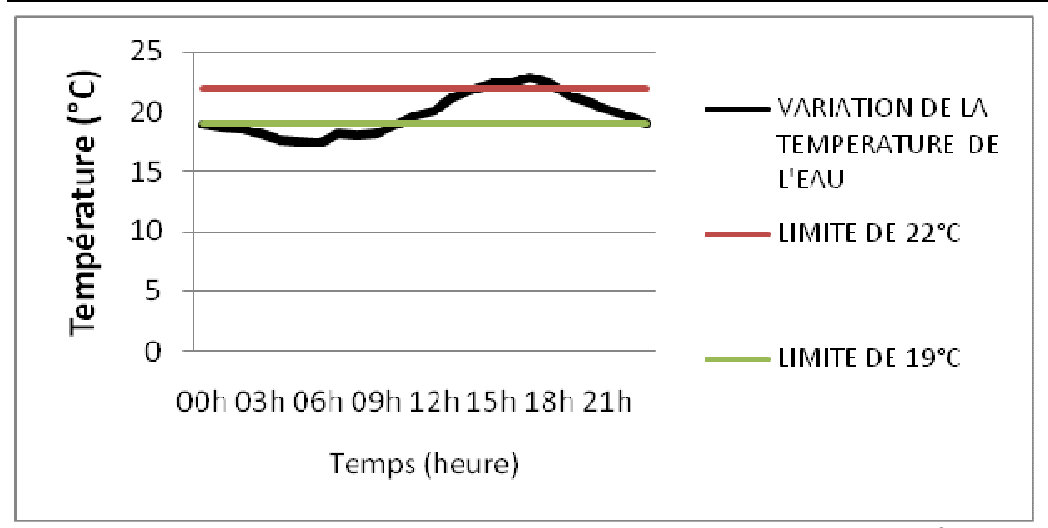

Figure 3 : Variation moyenne de température de l'eau de l'étang en fonction des heures

Variation de la température en fonction de la profondeur : L'évaluation de l'impact de la profondeur sur les températures de l'eau de l'étang (min, max et moyenne) montre que les températures à $15 \mathrm{~cm}$ et 100 $\mathrm{cm}$ de profondeur ne varient pas significativement
(Figures 4-6). Par contre, les températures ambiantes étaient trop basses pour les températures minimales $(p<0,05)$ et trop élevées pour les températures maximales $(p<0,05)$ et moyennes $(p<0,05)$.

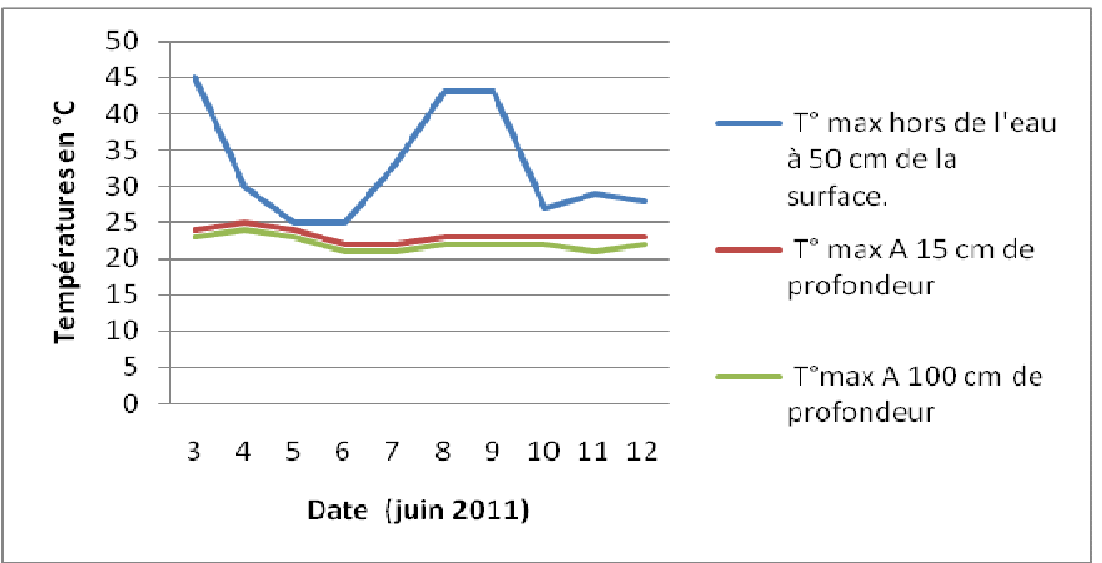

Figure 4 : Variation des températures maximales de l'eau dans l'étang

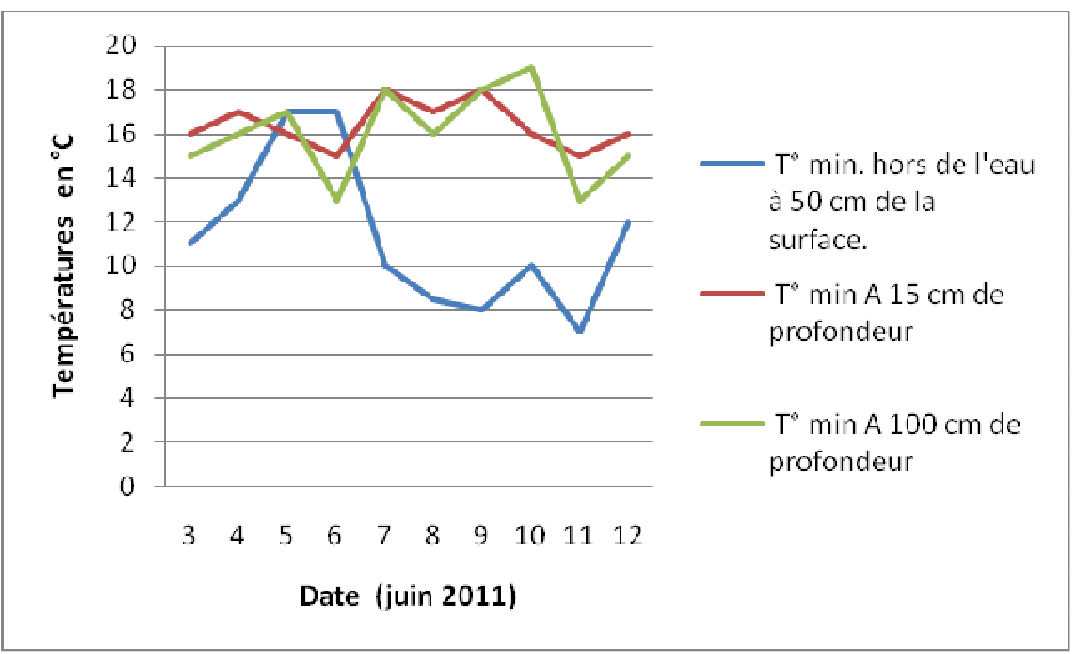

Figure 5 : Variation des températures minimales de l'eau dans l'étang 


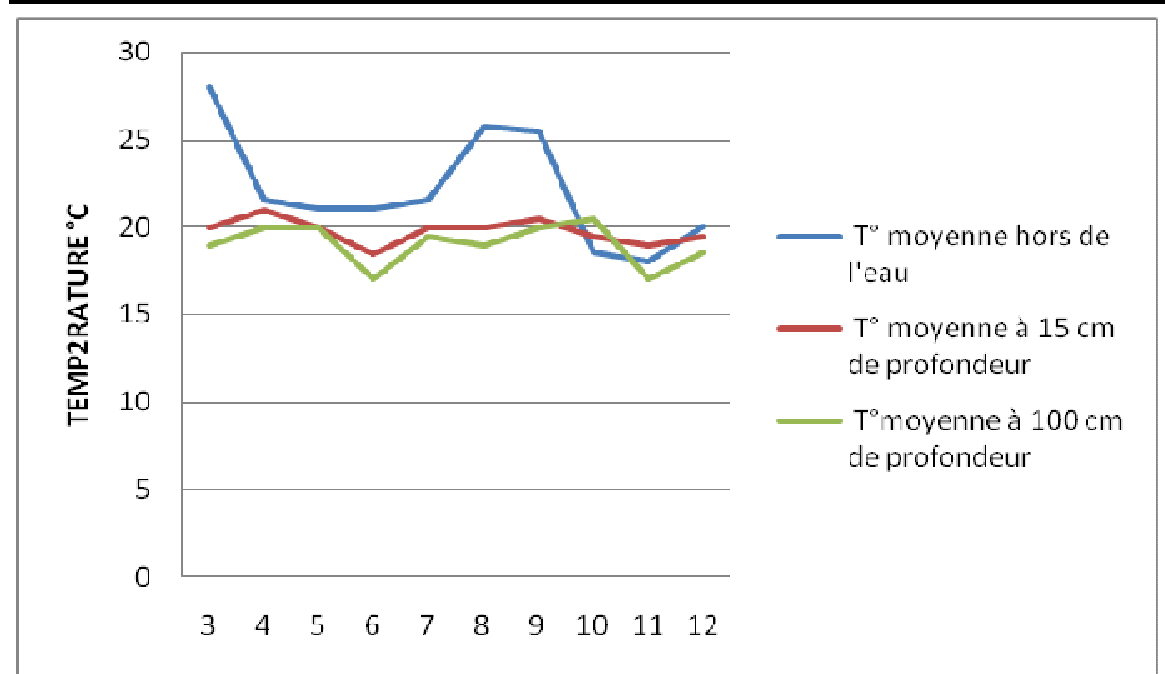

Figure 6 : Variation des températures moyennes de l'eau dans l'étang

\section{DISCUSSION}

Variation journalière de température de l'eau: La variation de la température durant 10 jours successifs, en période froide, a montré qu'il existe deux périodes des fluctuations importantes. Premièrement, la période allant de $22 \mathrm{~h}$ à 10 heures enregistre des basses températures de $17^{\circ} \mathrm{C}$ à $19^{\circ} \mathrm{C}$; la température la plus basse étant observée à 6 heures du matin. Cette courbe présente une similitude avec celle que Mukasikubwabo (1990) a obtenu au Rwanda pendant la période froide. Toutefois, les eaux de notre station sont plus froides que celle du Rwanda. En effet, Mukasikubwabo (1990) a enregistré à la station piscicole de Rwasave (Rwanda), durant la saison sèche, des variations de température de $19,6^{\circ} \mathrm{C}$ (à 7 heures le matin) à $28^{\circ} \mathrm{C}$ (à 16 heures). Dans les deux cas, une augmentation de température est observée peu après le lever du soleil. Deuxièmement, la température la plus élevée est atteinte à $17 \mathrm{~h}$ à la station de I'URA (Lubumbashi) et la baisse de température est observée après le coucher du soleil. II se dégage que les variations en hausse ou en baisse de la température de l'eau dans les étangs suivent la périodicité imprimée par le lever et le coucher du soleil. Ces résultats sont en conformité avec ceux obtenus par Boyd (1990), Coche et Muir $(1995 ; 1999)$ qui affirment que la variation de la température de l'eau dans les étangs est étroitement liée au rayonnement solaire. En somme, les écarts de températures observés dans nos travaux sont de $\pm 3^{\circ} \mathrm{C}$ en dessous de $22^{\circ} \mathrm{C}$ qui est le seuil minimum de référence (Balarin \& Haller, 1982). La température moyenne journalière des étangs pendant la période d'étude était de $19,4 \pm 2^{\circ} \mathrm{C}$. Cette valeur est en deçà de la fourchette de température optimale de $27^{\circ} \mathrm{C}$ à $31^{\circ} \mathrm{C}$ indiquée pour une production optimale d'Oreochromis niloticus (Mires, 1982 ; Kestemont et al., 1989 ; Kapetsky, 1996), espèce élevée sur notre site d'étude et abondamment produits dans les fermes piscicoles de la région de Lubumbashi. Ceci implique qu'en période froide à Lubumbashi, l'élevage d'O. niloticus est freinée par la baisse de température qui pourraient avoir des conséquences graves sur leur croissance et leur reproduction alors que la demande en poissons est en constante progression. En effet, ailleurs les études de Silvera (1978), Coche (1982), Guerrero \& Garcia, (1983), Hughes \& Behrends (1983), Parrel et al., (1986), Lovshin \& Ibrahim, (1987) et Azaza et al. (2010) ont révélé que de nombreux facteurs peuvent intervenir de façon significative sur la production des 0 . niloticus, mais la température est le facteur le plus important. Les poissons étant des animaux poïkilothermes, la température de l'eau affecte leur croissance et celle de leurs larves (Fontaine \& Le Bail, 2004). Ce facteur a des effets sur l'hétérogénéité de croissance observée dans les élevages. Ailleurs, d'autres études ont montré que la température d'élevage interagit avec les facteurs alimentaires sur les mécanismes impliqués dans la constitution des dépôts lipidiques (Corraze et al.,1999) et les basses températures s'accompagneraient de faible taux d'alimentation. Une élévation de la température de 17 à $23^{\circ} \mathrm{C}$ s'accompagne d'une augmentation de l'hétérogénéité de taille des juvéniles de perche commune et, en conséquence, du taux de cannibalisme (Kestemont \& Mélard, 2000). De plus, 


Lwamba et al. J. Appl. Biosci. Variations de la température de l'eau des étangs en période
froide à Lubumbashi (R.D. Congo) et implications pour la production des poissons

Paperna (1982) affirme qu'aux températures inférieures à $17^{\circ} \mathrm{C}$, les $\mathrm{O}$. niloticus diminuent leur activité et cessent de se nourrir alors qu'à $15^{\circ} \mathrm{C}$ (et en deçà), ces poissons sont affaiblis ou même stressés et deviennent sensibles aux maladies. El-Naggar et al., (2000) indiquent par ailleurs que le taux de ponte des géniteurs d'O. niloticus était nul à des températures inférieures à $19^{\circ} \mathrm{C}$ mais de l'ordre de 40 et $73 \%$ entre 22 à $27^{\circ} \mathrm{C}$. Au Cameroun, Nwamo et al. (2014) ont enregistré des taux de ponte nuls et de $86 \%$ à des températures respectives de $30-32^{\circ} \mathrm{C}$ et $28^{\circ} \mathrm{C}$, en utilisant l'espèce Penaeus kerathurus. En Égypte, la croissance de Macrobrachium rosenbergii est améliorée lorsque la température passe de 24 à $29^{\circ} \mathrm{C}$ mais est ralentie lorsque la température passe de 29 à $35^{\circ} \mathrm{C}$ (Habashy \& Hassan, 2011). Des résultats similaires ont été obtenus aux USA (Buckel et al., 1995). Ainsi, la moyenne de température obtenue au cours de nos travaux $\left(19,4 \pm 2^{\circ} \mathrm{C}\right)$ est largement en dessous des recommandations émises par les auteurs pour une production optimale des espèces telles que l'O. niloticus (Huet, 1972; Kestemont et al.,1989; Mires, 1982 ; Kapetsky, 1996).

Variation de la température en fonction de la profondeur : L'évaluation de l'impact de la profondeur sur les températures de l'eau de l'étang (min, max et moyenne) montre que les températures à $15 \mathrm{~cm}$ et 100

\section{CONCLUSION}

La mise en évidence des valeurs de température qui caractérisent les conditions défavorables en période froide à Lubumbashi était le principal objectif de ce travail Les valeurs de températures obtenues ont montré qu'il existe deux périodes des fluctuations importantes, la période allant de $22 \mathrm{~h}$ à 10 heures et celle allant de 11 heures à 21 heures. La moyenne journalière de la température de l'eau dans les étangs durant cette période était de $19,4 \pm 2^{\circ} \mathrm{C}$. II s'est dégagé une différence significative $(p<0,001)$ entre la température moyenne et le seuil recommandé dans la production piscicole dans nos milieux $\left(22^{\circ} \mathrm{C}\right)$. Par conséquent, en période froide à Lubumbashi, l'élevage d'O. niloticus serait freinée par la baisse de

\section{REMERCIEMENTS}

Les auteurs remercient le Professeur Gustave Beya Siku et son Excellence Moïse Katumbi Chapwe pour leur contribution significative à cette étude. Nous

\section{BIBLIOGRAPHIE}

Azaza M.S., Legendre M., Kraiem M.M. \& Baras E., $\mathrm{cm}$ sont similaires, mais inférieures à la température obtenue hors l'eau. Ces résultats sont en conformité avec ceux obtenus par Coche \& Muir $(1995$; 1999) qui affirment que la température de l'eau dans un étang peu profond (ne dépassant pas $1 \mathrm{~m}$ ) est homogène suite au brassage réalisé par vent. Par ailleurs, les températures ambiantes étaient trop basses pour les températures minimales et trop élevées pour les températures maximales et moyennes. Nos résultats sont en contradiction avec ceux obtenus par Boyd (1990) qui indique la température de l'eau est tributaire des températures de l'air. Par contre, Coche \& Muir (1995, 1999) confirmant en partie nos résultats, précisent que c'est seulement lorsque la température de l'air est proche de sa valeur minimale ou maximale que la corrélation existe entre la température de l'eau dans un étang et de l'air à $50 \mathrm{~cm}$ au dessus de la surface de l'eau. II importe donc de maitriser la variation de la température d'eau des étangs en période froide à Lubumbashi, car à coté des insuffisances dans la reproduction artificielle et la non maitrise de l'alimentation, Tabaro et al. (2005) indiquent que la non maitrise des conditions environnementales, notamment la température, conduit à ce que la plupart d'élevage des poissons n'atteint pas les résultats escomptés en Afrique.

température qui pourraient avoir des conséquences graves sur leur croissance et leur reproduction. Pourtant, avec sa croissance démographique élevée, la demande en poissons est en constante progression à Lubumbashi. Ainsi, aux cotés des contraintes liées à l'alimentation des poissons, les résultats de notre étude montrent qu'il importe d'évaluer la réponse des poissons aux variations de températures en période froide et de mettre en place des systèmes de chauffage d'étangs adaptés au faible revenu des pisciculteurs de la zone d'étude. Des résultats évaluant la réponse des poissons aux variations de température en période froide et l'efficacité des systèmes de chauffage d'étangs en pisciculture sont en cours.

remercions également le lecteur anonyme pour ses commentaires constructifs.

2010. Size-dependent effects of daily thermal 
fluctuations on the growth and size heterogeneity of Nile tilapia Oreochromis niloticus. Journal of Fish Biology, 76: 669-683.

Balarin J.D. \& Haller R.D., 1982. The intensive culture of tilapia in tanks, raceways and cages [En ligne]. Recent Advances in Aquaculture, vol. 1. Croom Helm, London. Http://www.fao.org/docrep

Balon E.K. \& Coche A.G., 1974. Lake Kariba : a manmade tropical ecosystem in Central Africa. Monogr. Biol. 24.W. Junk, The Hague.

Boyd C.E., 1990. Water quality in ponds for aquaculture. Front Cover. - Science - Alabama Agricultural Experiment Station, Auburn University, $482 \mathrm{p}$.

Bruton M.N. \& Allanson B.R., 1974. Growth of Tilapia mossambica (Pisces: Cichlidae) in Lake Sibaya, South Africa. J. Fish. Biol., 6 : 701715.

Buckel J.A., Steinberg N.D. \& Conover D.O., 1995. Effects of temperature, salinity and fish size on growth and consumption of juvenile bluefish. Journal of fish biology 47: 696-706

Caudron A., 2008. Étude pluridisciplinaire des populations de truite commune (Salmo trutta L.) des torrents haut-savoyards soumises à repeuplements : diversité intra-spécifique, évaluation de pratiques de gestion et ingénierie de la conservation de populations natives. Thèse de Doctorat, Université de Savoie, 295p.

Coche A.G., 1982. Cage culture of tilapias. In: Biology and culture of tilapias. Pullin R.S.V. and LoweMcConnell R.H. (éds.). ICLARM, Philippines, 205-246.

Coche A.G. \& Muir J.F., 1995. Pisciculture continental: la gestion. Les étangs et leur eau. Collection FAO, Formation. Volume 21. 328p.

Coche A.G., Muir J.F., 1999. Pisciculture continental: la gestion. La ferme et ses stoks. Collection FAO, Formation. Volume 21. $341 \mathrm{p}$.

Corraze G., Larroquet L. \& Médale F., 1999. Domestication et croissance chez les poissons. INRA Prod. Anim., 12 (4) : 249-256.

Daget J., 1952. Mémoire sur la biologie des poissons du Niger moyen. 1. Biologie et croissance des espèces du genre Alestes. Bull. Inst. fr. Afr. noire (A), 14 (1) : 191-225.

Daget J., 1956. Mémoire sur la biologie des poissons du Niger moyen : recherches sur Tilapia zillii (Gerv.). Bull. Inst. fr. Afr. noire (A), 18 (1) :
165-233.

De Villers J., Squilbin M. \& Yourassowsky C., 2005. Qualité physico-chimique et chimique des eaux de surface: cadre général. Les données de I'Institut Bruxellois pour la Gestion de I'Environnement / Observatoire des Données de l'Environnement: "L'eau à Bruxelles" Fiche 2. Novembre 2005. 16p.

Déqué M. \& Somot, S. 2008. Extreme precipitation and high resolution with Aladin. Idöjaras QJ Hung Meteorol Serv, 112(3-4) : 179-190.

Durand J.R., 1978. Biologie et dynamique des populations d'Alestes baremoze (pisces, characidae) du bassin Tchadien. Trav. Doc. Orstom, Paris 98. 322p.

El-Gamal A., 2009. Effects of temperature on hatching and larval development and mucin secretion in common carp, Cypinus carpio (Linnaeus 1758). Global veterinaria 3(2): 80-90

El-Naggar G.O., El Nady M.A., Kamar M.G. \& AlKobabay A.l., 2000. Effect of photoperiod, dietary protein and temperature on reproduction in Nile tilapia (Oreochromis niloticus). Fitzsimmons, $K$. and Filho, Jomar Carvalho: American Tilapia Association and ICLARM, 352-358.

Fontaine P. \& Le Bail P-Y., 2004. Domestication et croissance chez les poissons. INRA Prod. Anim., 17 (3): 217-225.

Guerrero R.D. \& Garcia A.M., 1983. Studies on the fry production of Sarotherodon niloticus in a lakebased hatchery, 388-393. In: Fishelson, L. et Yaron, S. Eds, The First International Symposium on Tilapia in Aquaculture, Nazareth, Israel,Tel Aviv University, 624p.

Habashy M.M. \& Hassan M.M.S., 2011. Effects of temperature and salinity on growth and reproduction of the freshwater prawn, Macrobrachium rosenbergii (Crustacea Decapoda) in Egypt. International journal of environnemental science and engineering 1: 83-90

Halwart M. \& Gupta, M.V. 2010. L'élevage de poisson en rizière. Rome, FAO et World Fish Center. $87 p$.

Hopson A.J., 1972. A study of the Nile Perch Lates niloticus L. (Pisces, Centropomidae) in Lake Tchad. Overseas Res. Publ., no 19.

Huet M., 1970. Traité de pisciculture. Ed. Ch. de Wyngaert; 1200 Bruxelles. $718 p$.

Huet M., 1972. Textbook of Fish Culture: Breeding and 
Cultivation of Fish. Fishing News Ltd., Farnham. Surrey, England, UK. 436p.

Hughes D.G. \& Behrends L.L., 1983. Mass production of Tilapia nilotica seed in suspended net enclosure, 394-401. In: Fishelson, L. et Yaron, S. Eds, The First International Symposium on Tilapia in Aquaculture, Nazareth, Israel May 813, 1983, Tel Aviv University, 624p.

Kapetsky J.M., 1996.Évaluation stratégique des possibilités de pisciculture en étang à température élevée sur le continent africain. Document technique du CPCA. No. 27. Rome, FAO. 77p.

Kestemont P., Micha J.C. \& Falter U., 1989. Les méthodes de production d'alevins de Tilapia nilotica. Project reports. ADCP/REP/89/46. http://www.fao.org - Archives de documents de la FAO.

Leblanc M. \& Malaisse F., 1978. Lubumbashi, un écosystème urbain tropical. Centre international de sémiologie, Université nationale du Zaïre.

Leblanc Y., Rosolen C., de la Noë J. \& Aubier M.G., 1978. Journées de planétologie, Edit. CNRS, 43p.

Lovshin L.L. \& Ibrahim H.H., 1987. Effects of broodstock exchange on Tilapia nilotica egg and fry production in net enclosures, 231-236. In: R.S.V. Pullin et al: The Second International Symposium on tilapia in Aquaculture. ICLARM Conference Proceedings 15, 623p.

Mires D., 1982. A Study of the Problems of the Mass Production of Hybrid Tilapia Fry. In R.S.V. Pullin and R.H. Lowe-McConnell (Eds) The biology and culture of tilapias, ICLARM Conference Proceedings 7, 432p. Int. Center for Living Aquatic Res. Management, Manila, Philippines: 317-329.

Moreau J., 1979. Biologie et évolution des peuplements de Cichlidés (Pisces) introduits dans les lacs malgaches d'altitude. Thèse Doctorat d'État, I.N.P. Toulouse, $345 p$.

Mukasikubwabo V., 1990. Contrôle de l'ovogénèse chez Tilapia nilotica : effets de la température et de l'illumination nocturne. Mémoire, Université de Québec, $115 p$.

Nwamo R.D., Kenfack T.C., Ajonina G., Tomedi E.M. \& Dibong S.D., 2014. Effets de la salinité et de la temperature sur le taux d'éclosion des oeufs de Pennaeus kerathurus (Kribi, Cameroun).
Journal of animal and plant science 23(1): 3510-3520

Omasaki S. K., Charo-Karisa H.\& Kosgey I. S., 2013. Fish production practices of smallholder farmers in western Kenya. Livestock Research for Rural Development. Volume 25, Article \#52. Retrieved April 8, 2013, from http://www.Irrd.org//lrd25/3/omas25052.htm

Paperna I., 1982. Parasites, infections et maladies du poisson en Afrique. CPCA,Doc. Tech., (7):202 p.

Parks R.W., Scarsbrook E. \& Boyd C.E. 1975. Phytoplankton and water quality in fertilized fish pond. Alabama Agricultural Experiment Station, Auburn University. Ala. Cir. 224. 16 p.

Parrel P., Ali I. \& Lazard J., $1986 . \quad$ Le développement de l'aquaculture au Niger: Un exemple d'élevage de Tilapia en zone sahélienne. Bois et Forêts des Tropiques, $212: 71-94$.

Silvera P.A.W., 1978. Factors affecting fry production in Sarotherodon niloticus (L.), M.Sc. thesis, Auburn University, Auburn, Alabama, 1p.

Spore, 2013. Aquaculture: un bel avenir s'ouvre en Afrique. http://sporecta.int

Tabaro R.S., Micha J-C., Ducarme C., 2005. Essais d'adaptation de production massive de juvéniles de Clarias gariepinus en conditions rurales. Tropicultura 23(4) : 231-244

Valeta J.S., Likongwe S.J., Kassan D. \& Maluwa A.O., 2013. Temperature-dependent egg development rates, hatchability and fry survival rate of lake Malawi Tilapia (Chambo), Oreochromis karongue (Pisces: cichlidae). International journal of fisheries and aquaculture 5(4): 55-59 